Published in final edited form as:

Curr Opin Ophthalmol. 2013 May ; 24(3): 239-243. doi:10.1097/ICU.0b013e32835f8e55.

\title{
Update on Depression and Age-Related Macular Degeneration
}

\author{
Robin J. Casten, PhD and \\ Jefferson Medical College, Department of Psychiatry and Human Behavior \\ Barry W. Rovner, MD \\ Jefferson Medical College, Departments of Neurology and Psychiatry
}

\begin{abstract}
Introduction-This review updates the literature on depression in age-related macular degeneration.
\end{abstract}

\begin{abstract}
Purpose of review-Treatment for AMD has been revolutionized since the 2004 review of depression and AMD. New data describing the prevalence of depression in AMD, as well as novel interventions for managing depression in AMD, are discussed.
\end{abstract}

Recent findings-Depression continues to be prevalent in AMD and new information is available on the pathways by which impaired vision leads to depression. Strategies for the treatment of depression in patients with impaired vision have evolved.

Summary-AMD is still a major risk factor for depression and people with activity restriction due to vision loss are at greatest risk. An integrated approach to depression management in older adults with impaired vision may be the best course of action.

\section{Keywords}

AMD; depression; late-onset vision loss

\section{Introduction}

Depression in people aged 65 and older is a serious yet often under diagnosed and under treated medical condition. The prevalence of depression among community dwelling older people is estimated to be approximately $12 \%$ (for both major and minor depression combined). ${ }^{[1]}$ Risk factors include female gender, medical comorbidity, functional loss, sleep disturbance, and a previous history of depression. ${ }^{[2]}$ Vision impairment almost doubles the risk of depression. ${ }^{[3,4]}$ The prevalence of depression among older persons with impaired vision ranges from about $14 \%$ to $63 \%$. [5-7]

In 2004 we reviewed the literature on depression in age-related macular degeneration (AMD), which is the most common cause of vision loss in older persons. ${ }^{[8]}$ The prevailing themes were the reduced quality of life, the high prevalence of depression and its disabling

Corresponding Author: Robin Casten, PhD, Thomas Jefferson University, Jefferson Hospital for Neuroscience, 900 Walnut Street, $2^{\text {nd }}$ floor, Philadelphia, PA 19107, 215-503-1250, Robin.Casten@jefferson.edu.

No conflicts of interests have been declared. 
effects, independent of severity of vision loss. ${ }^{[9]}$ Older people with impaired vision also face greater risks of falls, hip fracture, nursing home placement, and death. ${ }^{[10,11]}$ Proposed mechanisms linking AMD to depression include impaired function and the loss of the ability to pursue valued activities. In 2004 there were few evidence-based treatments for remediating and/or preventing depression in AMD and its concomitant functional impairment.

In the past decade effective treatments for neovascular disease have been developed, tested, and integrated into usual care. The level of success achieved with anti-VEGF treatment in NV-AMD is unprecedented. Rarely do we see such an incredibly debilitating disease treated so effectively. This updated review will discuss depression in the context of this revolutionary treatment modality, including a description of studies that elucidate the mechanisms that link AMD and depression, and that have tested innovative interventions to treat depression in AMD.

The efficacy of ranibizumab and bevacizumab to improve and maintain vision is wellestablished. Approximately one third of patients with neovascular AMD gain at least 15 letters by 12 months ${ }^{[12]}$ Patients also improve in vision function and quality of life. ${ }^{[13,14]}$ For example, in the MARINA trial, subjects in the two active treatment arms (ranibizumab $0.3 \mathrm{mg}$ and ranibizumab $0.5 \mathrm{mg}$ ) had significant improvements in the 4 NEI-VFQ subscales that relate to psychosocial functioning (i.e., mental health, role difficulties, dependency, social functioning) whereas control subjects declined. But despite these important treatment effects, a significant portion of patients receiving active treatment did not exhibit substantial improvements in vision (i.e., did not gain $\geq 15$ letters), and slightly more than $10 \%$ lost at least 5 letters after 1 year of treatment. About half failed to achieve a meaningful improvement in vision function. ${ }^{[15]}$ AMD patients, therefore, continue to be at risk for depression.

Newer research confirms that depression is still prevalent among AMD patients. A recent pilot study of 51 AMD patients receiving anti-VEGF treatment showed that 20\% were depressed 3 months after beginning anti-VEGF injections. Compared to nondepressed patients, the depressed patients were more likely to have had a decline in vision. ${ }^{[16]}$ This study suggests that patients who fail to achieve optimal treatment benefits from anti-VEGF injections or whose treatment outcomes fall below their expectations may be at an increased risk for depression. While vision restoration in these patients may be limited, a major contributor to poor vision function, namely depression, can nevertheless be treated.

Even patients with minimal vision loss are at risk for depression. In a multisite study of 764 low vision patients from 28 clinics across the US (most of whom had AMD), 22\% reported feeling depressed and $23 \%$ indicated that they were experiencing anxiety. What is remarkable is that over one third of these low vision clinic patients had only mild vision loss (best eye acuity 20/20 to 20/60) yet they considered their compromised vision function serious enough to warrant rehabilitation..$^{\left[17^{* *}\right]}$ This study adds to an abundance of evidence that shows the salience of impaired function over degree of vision loss on quality of life and depression. ${ }^{[18]}$ 
Bookwala et al. ${ }^{\left[19^{* *}\right]}$ explored this phenomenon in an in-depth study that delineated pathways linking impaired vision to depression in older people. Results showed that physical limitations and social isolation are the mechanisms by which older people with impaired vision develop depression. Interestingly, driving limitation was not a contributor to the vision impairment/depression relationship. Similar results were seen in a recent study by Mathew et al. (2011). ${ }^{\left[20^{* *}\right]}$ The investigators compared depressive symptoms and function in 145 patients with AMD and a control group matched for age and sex. The rate of clinically significant depression was $44 \%$ in the AMD group versus $18 \%$ in the control group. This disparity persisted even when controlling for medical comorbidities. Like the Bookwala et al. study, Mathew et al. showed that AMD patients become depressed when they experience reduced health and social functioning. These relationships were confirmed in yet another study. Rees et al. (2010) examined a group of 143 older low vision patients, over half of whom had AMD. Forty-two percent had significant depressive symptoms. Multivariate analyses indicated that patients reporting vision-specific distress were more prone to depression, particularly when they experienced activity restrictions. ${ }^{[21]}$

The prevalence of depression is also high among patients with other ophthalmologic diseases. Popescu et al. compared rates of depression in patients with AMD, glaucoma, Fuchs corneal dystrophy, and a control group with no ophthalmic disease. ${ }^{\left[22^{*}\right]}$ All 3 disease groups exhibited high depression rates (29\% to 39\%), and the rate was highest in the AMD group. Two thirds of the depressed subjects (across all 3 disease groups) indicated that they have limited their activities due to a fear of falling, and subjects with mobility restrictions were more likely to be depressed.

The evidence is overwhelming that AMD remains a significant risk factor for depression, and that patients who experience activity limitations or functional impairments are particularly vulnerable. The current preferred practice guidelines from the American Academy of Ophthalmology recognize that depression is a common comorbid condition of AMD and recommend screening and providing appropriate referrals. ${ }^{[23]}$ However, ophthalmologists often underestimate the negative effect of AMD on patients' quality of life, particularly among patients with better acuities. ${ }^{[24]}$ Consequently, depression in older adults with vision loss is often not detected and is undertreated. For example, Rees et al (2010) found that only $20 \%$ of depressed patients with impaired vision were being treated for their depression. ${ }^{[21]}$ Moreover, research examining relationships between depression and severity of vision loss is mixed. Some studies show no relationship, ${ }^{[25,26]}$ while others indicate that depression severity is worse in those with more substantial vision loss. ${ }^{[27]}$ Until this uncertainty is resolved, it may be best to screen all patients with AMD for depression, regardless of their acuity, disease stage, or anticipated treatment, especially because patients who are disappointed in their treatment outcomes may be at increased risk.

A major barrier in screening for depression is limited understanding of depression. ${ }^{\left[28^{*}\right]}$ Rees at al. interviewed 94 eye care staff (including 20 ophthalmologists) regarding their attitudes towards and knowledge about depression. On average the respondents estimated that slightly more than half of all ophthalmology patients may be depressed, and the majority was able to correctly identify symptoms of depression. Nevertheless, many failed to recognize the physical symptoms of depression (e.g., body pain) and incorrectly believed that depression 
could be treated with sleep medications or alcohol. Respondents' confidence levels regarding discussing depression and depression treatment were moderate at best; those who had received prior depression training expressed greater confidence.

In response to these survey results, Rees et al. developed and pilot tested a depression training program for eye health professionals. ${ }^{[29]}$ The program was delivered over the course of three 90-minute sessions, and covered topics such as education about depression, screening methods for depression, and strategies for implementing screening and referral mechanisms into clinical care. Post-training interviews showed that confidence in discussing depression and depression treatment increased. Moreover, after the training participants indicated that they would be more likely to screen patients for depression and refer them for treatment. Forty-four percent of participants have initiated plans for depression screening in their practices after participating in the training program.

Depression is usually treated with medication and/or psychotherapy. In recent years behavioral and self-management programs for AMD patients have been developed and tested in randomized controlled trials. Brody et al.'s seminal study evaluated the benefits of a self-management intervention in patients with AMD. Their 6-week program consisted of didactic instruction regarding basic information about AMD and rehabilitative strategies. It also contained a behavioral component to help patients handle some of the challenges faced by people with AMD. The intervention group evidenced a significant improvement in mood 6 weeks post baseline and that this effect was most pronounced for those who were depressed at baseline. Intervention subjects also displayed improved function, and subjects who were depressed at baseline in particular benefited from the intervention. A follow-up study at 6 months demonstrated the intervention's sustained effects. The benefits were greatest for subjects who had been depressed at baseline. Importantly, the incidence of depression from baseline to 6 months was significantly lower in the intervention group. $[30,31]$

The "Preventing Depression in AMD" trial tested the efficacy of a psychosocial intervention, Problem Solving Treatment (PST), to prevent depression in patients with new onset vision loss due to AMD. This standardized intervention was based on the premise that inaccurate appraisals of problems and dysfunctional problem solving skills contribute to the onset of depression. Teaching patients effective problem solving skills, therefore, may foster independence, preserve function, and prevent depressive symptoms. This study enrolled 206 nondepressed patients with bilateral neovascular AMD and recent vision loss. Subjects were randomized to either PST or a usual care control condition. The primary outcome was incidence of depressive disorders at 2 months (short-term effect); the trial also evaluated effects at 6 months (maintenance effect). A secondary goal was to determine whether PST could prevent the loss of participation in valued activities. PST-treated subjects had 6 inhome sessions in which a specially trained interventionist taught subjects a systematic stepby-step process for dealing with vision-related problems (for example, trouble cooking, difficulty reading). Results indicated that PST successfully prevented depression at 2 months. About $12 \%$ of the PST group became depressed compared to $23 \%$ of the usual care group $(\mathrm{p}<.05)$. There was a parallel effect for preserving functional abilities. Subjects treated with PST were less likely to relinquish valued activities than usual care subjects 
( $23 \%$ versus $37 \%$, respectively; $\mathrm{p} \leq 0.04$ ). A meditational analysis determined that PST prevented depression to the extent that activity relinquishment was minimized. These effects, however, were not sustained, as rates of depression were similar in both groups at 6-months (21\% of PST subjects developed depression vs. $27 \%$ of control subjects). ${ }^{[32]}$

This trial demonstrated that depression can be prevented in the short term; however, beneficial effects decay over time. Because neovascular AMD is characterized by episodes of sudden vision loss and corresponding functional declines, ongoing efforts to prevent depression may be needed throughout the course of the disease. This may involve monitoring and screening for events that may trigger depression and then administering maintenance or booster treatments as needed.

Another strategy for treating and/or preventing depression is to bundle depression treatment with low vision rehabilitation. Support for this approach was demonstrated in a study which examined a group of older people newly referred for low vision rehabilitation. Results showed that active participation in rehabilitation was related to a decrease in depressive symptoms over a two-year period. ${ }^{[33]}$ Girdler et al. compared the benefits of an enhanced model of low vision rehabilitation with a usual care model in 77 older persons attending a low vision clinic (most had AMD). The enhanced intervention was an 8-week group program and was led by a social worker and an occupational therapist. Subjects were taught strategies for managing their vision loss. Subjects in the enhanced intervention reported higher levels of activity engagement. At 12 weeks follow-up, fewer subjects in the enhanced intervention reported depressive symptoms compared to controls ( $17 \%$ vs. $51 \%$; $\mathrm{p} \leq 0.001$ ), and a greater proportion demonstrated improvements in activity participation, self-efficacy, and adjustment to vision loss. ${ }^{[34]}$ This study shows that an integrated low vision rehabilitation that addresses psychosocial functioning may prevent and treat depression.

Two clinical trials are the process of testing the efficacy of comprehensive approaches that simultaneously address impaired vision and depression. Margrain et al. is looking at an integrated approach in which low vision patients will be screened for depression. Patients who have significant depressive symptoms will be randomized to a psychosocial intervention arm, a depression referral group, or a wait list control group. Subjects in the active treatment group will receive a collaborative problem solving treatment in which a trained interventionist will provide depression treatment and will integrate the low vision treatment plans into the patients' care.[35]

Rovner and Casten are in the final stages of a clinical trial to test the efficacy of a depression-focused low vision rehabilitation intervention to prevent depression in high risk patients with AMD. Two hundred AMD patients with subthreshold depressive symptoms are evaluated by a low vision optometrist, prescribed appropriate low vision devices, and are randomized to 6 in-home occupational therapy sessions or 6 in-home placebo sessions that involve supportive therapy (i.e., empathic listening). Subjects in the occupational therapy group, in addition to being taught compensatory strategies, are administered a behavioral treatment for depression. The primary outcome is depression status at 4 months: it is hypothesized that this comprehensive intervention will prevent depressive disorder in high risk patients with AMD. 
On a final note, from a research perspective the effect of depression on subsidiary clinical trial outcomes such as quality of life and vision function warrants comment. Depressed patients tend to underestimate their functional capabilities, and thus self-ratings on instruments such as the NEI-VFQ may reflect both actual abilities as well as negative reporting biases. ${ }^{[36]}$ For this reason, depression should be assessed and controlled for in clinical trials of AMD treatment. Failure to recognize and control for this important influence may lead to underestimates of treatment impact on patients' lives.

\section{Conclusion}

AMD is a serious public health problem, robbing patients of independence and degrading their quality of life. As the population ages, greater numbers of Americans will be affected by impaired vision as well as subsequent depression. Thus targeting depression, a major contributor to vision-related disability, is one strategy for maintaining independence and improving quality of life. Moreover, because depression and function are so closely intertwined in AMD, developing interventions that incorporate depression management into rehabilitation might be the best course of action. Anti-VEGF treatment has allowed us to make tremendous strides in improving a major source of vision disability in AMD. Addressing the psychological factors will allow us to even further reduce disability, and thereby improve quality of life.

\section{Acknowledgments}

This work was supported by grant U01 5U01EY018819 from the National Eye Institute.

\section{References}

1. Steffens D, Fisher G, Langa K, Potter G, Plassman B. Prevalence of Depression among older Americans: the Aging, Demographics and Memory Study. International Psychogeriatrics. 2009; 21:879-888. [PubMed: 19519984]

2. Cole M. Evidence-Based Review of Risk Factors for Geriatric Depression and Brief Preventive Interventions. Psychiatr Clin N Am. 2005; 28:785-803.

3. Nyman S, Gosney M, Victor C. The psychosocial impact of sight loss: An Update of the evidence. Age and Ageing. 2009; 38:ii65.

4. Huang C, Dong B, Lu Z, Yue J, Liu Q. Chronic diseases and risk for depression in old age: A metaanalysis of published literature. Ageing Research Reviews. 2010; 9:131-141. [PubMed: 19524072]

5. Evans J, Fletcher A, Wormald R. Depression and Anxiety in Visually Impaired Older People. Ophthalmology. 2007; 114:283-288. [PubMed: 17270678]

6. Jones G, Rovner B, Crews J, Danielson M. Effects of Depressive Symptoms on Health Behavior Practices Among Older Adults With Vision Loss. Rehabilitation Psychology. 2009; 54:64-172.

7. Rovner B, Ganguli M. Depression and disability associated with impaired vision: the MoVies Project. J Am Geriatr Soc. 1998; 46:617-619. [PubMed: 9588377]

8. Congdon N, O'Colmain B, Klaver C, et al. Causes and prevalence of vision impairment among adults in the United States. Arch Ophthalmol. 2004; 122:477-485. [PubMed: 15078664]

9. Casten R, Rovner B, Tasman W. Age-related macular degeneration and depression: A review of recent research. Current Opinions in Ophthalmology. 2004; 15:181-183.

10. Wysong A, Lee P, Sloan F. Longitudinal Incidence of Adverse Outcomes of Age-Related Macular Degeneration. Arch Ophthalmol. 2009; 127:320-327. [PubMed: 19273797] 
11. Tournier M, Moride Y, Ducruet T, et al. Depression and mortality in the visually-impaired, community-dwelling, elderly population of Quebec. Acta Ophthalmologica. 2008; 86:196-201. [PubMed: 17888085]

12. The CATT Research Group. Ranibizumab and Bevacizumab for Neovascular Age-Related Macular Degeneration. NEJM. 2011; 364:1897-908. [PubMed: 21526923]

13. Rosenfeld P, Brown D, Heier J. Ranibizumab for Neovascular Age-Related Macular Degeneration. NEJM. 2006; 355:1419-1431. [PubMed: 17021318]

14. Brown D, Kaiser P, Michels M, et al. Ranibizumab versus Verteporfin for Neovascular AgeRelated Macular Degeneration. NEJM. 2006; 355:1432-1444. [PubMed: 17021319]

15. Chang T, Bressler N, Fine J, et al. Improved Vision-Related Function After Ranibizumab Treatment of Neovascular Age-Related Macular Degeneration: Results of a Randomized Clinical Trial. Arch Ophthalmol. 2007; 125:1460-1469. [PubMed: 17998507]

16. Casten R, Rovner B, Leiby B, Tasman W. Depression despite Anti-VEGF Treatment of AMD. Archives of Ophthalmology. 2010; 128:506-507. [PubMed: 20385955]

**17. Goldstein J, Massof R, Deremeik J, et al. Baseline Traits of Low Vision Patients Served by Private Outpatient Clinical Centers in the United States. Arch Ophthalmol. 2012; 130:10281037. This study describes psychosocial and functional characteristics of people seeking low vision services, many of whom have AMD. [PubMed: 22893074]

18. Zhang X, Bullard K, Cotch M, et al. Depression and functional vision loss are associated in persons aged 20+ years in the United States, NHANES 2005-2008. Arch Ophthalmol. in press.

**19. Bookwala J, Lawson B. Poor Vision, Functioning, and Depressive Symptoms: A Test of the Activity Restriction Model. The Gerontologist. 2011; 51:798-808. This study demonstrates that social isolation and physical limitations are the pathways by which impaired vision leads to depression. [PubMed: 21737397]

**20. Mathew R, Delbaere K, Lord S, Beaumont P. Depressive symptoms and quality of life in people with age- related macular degeneration. Ophthalmic \& Physiological Optics. 2011; 31:375-380. This study documents the high rate of depression in AMD and demonstrates the role of functional limitations in the development of depression. [PubMed: 21679317]

21. Rees G, Tee H, Marella M, et al. Vision-specific distress and depressive symptoms in people with vision impairment. IOVS. 2010; 51:2891-2896.

*22. Popescu M, Boisjoly H, Schmaltz H, et al. Explaining the relationship between three eye diseases and depressive symptoms in older adults. Invest Ophthalmol Vis Sci. 2012; 53:2308-2313. This study compared rates of depression in subjects with 3 eye diseases and found that AMD subjects had the highest rate, and that depression was related to mobility restrictions. [PubMed: 22427589]

23. American Academy of Ophthalmology Retina/Vitreous Panel. Age-related macular degeneration. San Francisco (CA): American Academy of Ophthalmology (AAO); 2008. Available at www.AAO.org/ppp

24. Brown G, Brown M. Let us wake the nation on the treatment for age-related macular degeneration. Curr Opin Ophthalmol. 2010; 21:169-171. [PubMed: 20397317]

25. Rovner B, Casten R, Tasman W. Effect of depression on vision function in age-related macular degeneration. Arch of Ophthalmol. 2002; 120:1041-1044. [PubMed: 12149057]

26. Rover B, Casten R. Stability of visual acuity measurement in depression. Am J Geriatr Psychiatry. 2005; 13:255-258. [PubMed: 15728758]

27. Augustin A, Sahel J, Bandello F, et al. Anxiety and depression prevalence rates in age-related macular degeneration. Invest Ophthalmol Vis Sci. 2007; 48:1498-1503. [PubMed: 17389477]

*28. Rees R, Fenwick E, Keeffe J, et al. Managing depression in patients with vision impairment: A descriptive study of practitioners' beliefs and confidence. Australasian Journal on Ageing. 2011; 30:130-135. This descriptive study highlights the limited understanding of depression among eye health professionals. [PubMed: 21923706]

29. Rees G, Mellor D, Heenan H, et al. Depression Training Program for Eye Health and Rehabilitation Professionals. Optometry and Vision Science. 2010; 87:494-500. [PubMed: 20473238] 
30. Brody B, Gamst A, Williams R, et al. Depression, visual acuity, comorbidity, and disability associated with age-related macular degeneration. Ophthalmology. 2001; 108:1893-1900. [PubMed: 11581068]

31. Brody B, Roch-Levecq A, Gamst A, et al. Self-management of age-related macular degeneration and quality of life: a randomized controlled trial. Arch Ophthalmol. 2002; 120:1477-1483. [PubMed: 12427060]

32. Rovner B, Casten R, Hegel M, et al. Preventing depression in age-related Macular degeneration. Arch Gen Psychiatry. 2007; 64:886-892. [PubMed: 17679633]

33. Horowitz A, Reinhardt J, Boerner K. The effect of rehabilitation on depression among visually disabled older adults. Aging Ment Health. 2005; 9:563-570. [PubMed: 16214704]

34. Girdler S, Boldy D, Dhaliwal S, et al. Vision self-management for older adults: a randomised controlled trial. Br J Ophthalmol. 2010; 94:223-228. [PubMed: 20139291]

35. Margrain T, Nollett C, Shearn J, et al. The Depression in Visual Impairment Trial (DEPVIT): trial design and protocol. BMC Psychiatry. 2012; 12:1-8. [PubMed: 22230388]

36. Owsley C, McGwin G. Depression and the 25-Item National Eye Institute Visual Function Questionnaire in older adults. Ophthalmology. 2004; 111:2259-2264. [PubMed: 15582083] 


\section{Key Points}

1. Rates of depression in AMD are high even among patients who received antiVEGF treatment.

2. AMD patients who have vision-related activity limitations are at greatest risk for depression.

3. New treatment approaches that integrate depression management into low vision care are being developed. 